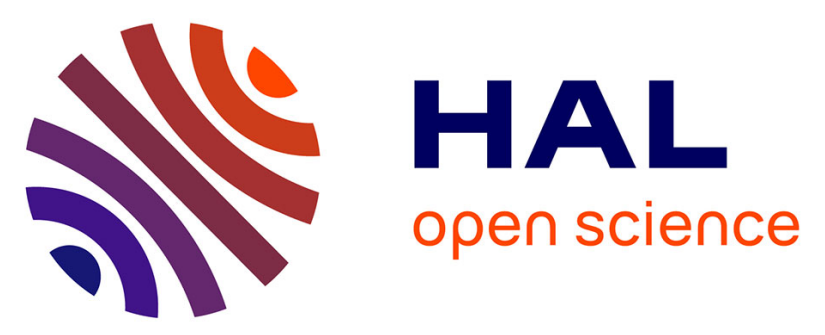

\title{
A Clear Correlation between the Diradical Character of 1,3-Dipoles and Their Reactivity toward Ethylene or Acetylene
}

\author{
Benoît Braïda, Christof Walter, Bernd Engels, Philippe C. Hiberty
}

\section{- To cite this version:}

Benoît Braïda, Christof Walter, Bernd Engels, Philippe C. Hiberty. A Clear Correlation between the Diradical Character of 1,3-Dipoles and Their Reactivity toward Ethylene or Acetylene. Journal of the American Chemical Society, 2010, 132 (22), pp.7631-7637. 10.1021/ja100512d . hal-01627884

\section{HAL Id: hal-01627884 \\ https://hal.science/hal-01627884}

Submitted on 9 Nov 2017

HAL is a multi-disciplinary open access archive for the deposit and dissemination of scientific research documents, whether they are published or not. The documents may come from teaching and research institutions in France or abroad, or from public or private research centers.
L'archive ouverte pluridisciplinaire HAL, est destinée au dépôt et à la diffusion de documents scientifiques de niveau recherche, publiés ou non, émanant des établissements d'enseignement et de recherche français ou étrangers, des laboratoires publics ou privés. 


\title{
A Clear Correlation between the Diradical Character of 1,3-Dipoles and Their Reactivity toward Ethylene or Acetylene
}

\author{
Benoit Braida, ${ }^{,+}{ }^{\dagger}$ Christof Walter, ${ }^{\ddagger}$ Bernd Engels, ${ }^{\ddagger}$ and Philippe C. Hiberty ${ }^{*,}$ \\ CNRS UMR 7616, Laboratoire de Chimie Théorique, UPMC Université Paris 06, Case \\ Courrier 137, 4 Place Jussieu, 75252 Paris, France, Institut für Organische Chemie, Am \\ Hubland, 97074 Würzburg, Germany, and Laboratoire de Chimie Physique, Bât 349, \\ Université de Paris-Sud, CNRS UMR 8000, 91405 Orsay, France
}

E-mail: braida@Ict.jussieu.fr; philippe.hiberty@u-psud.fr

\begin{abstract}
A series of nine 1,3-dipoles, belonging to the families of diazonium betaines, nitrilium betaines, and azomethine betaines, has been studied by means of the breathing-orbital valence bond ab initio method. Each 1,3-dipole is described as a linear combination of three valence bond structures, two zwitterions and one diradical, for which the weights in the total wave function can be quantitatively estimated. In agreement with an early proposition of Harcourt, the diradical character of 1,3-dipoles is shown to be a critical feature to favor 1,3-dipolar cycloaddition. Within each family, a linear relationship is evidenced between the weight of the diradical structure in the 1,3-dipole and the barrier to cycloaddition to ethylene or acetylene, with correlation coefficients of $0.98-1.00$. The barrier heights also correlate very well with the transition energies from ground state to pure diradical states of the 1,3-dipoles at equilibrium geometry. Moreover, the weight of the diradical structure is shown to increase significantly in all 1,3-dipoles from their equilibrium geometries to their distorted geometries in the transition states. A mechanism for 1,3-dipolar cycloaddition is proposed, in which the 1,3-dipole first distorts so as to reach a reactive state that possesses some critical diradical character and then adds to the dipolarophile with little or no barrier. This mechanism is in line with the recently proposed distortion/interaction energy model of Ess and Houk and their finding that the barrier heights for the cycloaddition of a given 1,3-dipole to ethylene and acetylene are nearly the same, despite the exothermicity difference (Ess, D. H.; Houk, K. N. J. Am. Chem. Soc. 2008, 130, 10187).
\end{abstract}

\section{Introduction}

1,3-Dipolar cycloaddition is a general and powerful method for the synthesis of five-membered heterocyclic compounds, owing to the great variety and availability of dipoles and dipolarophiles. ${ }^{1}$ According to the classification of Huisgen, ${ }^{1} 1,3-$ dipoles are classified in two general categories: bent dipoles of allylic type, which can be represented as $\mathrm{X}=\mathrm{Y}^{+}-\mathrm{Z}^{-}$, and linear dipoles of propargyl type, which have an additional double bond, $\mathrm{X} \equiv \mathrm{Y}^{+}-\mathrm{Z}^{-}$(or ${ }^{-} \mathrm{X}=\mathrm{Y}^{+}=\mathrm{Z}$ ). Actually, a more realistic representation of 1,3-dipoles must involve three Lewis structures, two zwitterionic ones and a diradical one, as is detailed in Scheme 1.

Renewed interest in 1,3-dipolar cycloaddition reactivity has recently been stimulated by some high-accuracy quantum mechanical calculations using the CBS-QB3 multicomponent method. In a theoretical study of 1,3-dipole cycloadditions involving three of the most important classes of 1,3-dipoles, namely the diazonium betaines $\mathrm{N} \equiv \mathrm{N}^{+}-\mathrm{Z}^{-}$, nitrilium betaines $\mathrm{HC} \equiv \mathrm{N}^{+}-\mathrm{Z}^{-}$, and azomethine betaines $\mathrm{H}_{2} \mathrm{C}=\mathrm{NH}^{+}-\mathrm{Z}^{-}(\mathrm{Z}=$

\footnotetext{
† UPMC Université Paris 06.

Institut für Organische Chemie.

${ }^{\S}$ Université de Paris-Sud.

(1) Synthetic Applications of 1,3-Dipolar Cycloaddition Chemistry Toward Heterocycles and Natural Products; Padwa, A., Pearson, W. H., Eds.; Wiley: New York, 2002.

(2) Ess, D. H.; Houk, K. N. J. Am. Chem. Soc. 2007, 129, 10646.

(3) Ess, D. H.; Houk, K. N. J. Am. Chem. Soc. 2008, 130, 10187.
}

$\mathrm{O}, \mathrm{NH}, \mathrm{CH}_{2}$ in each class), Ess and Houk provided accurate reaction barriers for cycloadditions of these nine dipoles to ethylene and acetylene., ${ }^{2,3}$

In agreement with qualitative predictions of frontier molecular orbital (FMO) theory, ${ }^{4}$ these authors found a monotonic decrease in barrier height from oxides to imines to ylides, as expected from the smooth decrease of HOMO-LUMO gaps in the same series of 1,3-dipoles. ${ }^{3}$ There was, however, an exception with nitrilium ylide, whose barriers to cycloadditions to both ethylene and acetylene were found to be $6 \mathrm{kcal} / \mathrm{mol}$ higher than those predicted by the FMO model. On the other hand, quite surprising and thought-provoking was the finding that barrier heights for the cycloaddition of a given 1,3-dipole to ethylene and acetylene are nearly the same, within $1.5 \mathrm{kcal} / \mathrm{mol}$, despite the exothermicity difference of ca. $16 \mathrm{kcal} / \mathrm{mol}$ in the general case, and even reaching $38 \mathrm{kcal} / \mathrm{mol}$ when aromatic cycloadducts are formed. This result, which is in complete contradiction with the reactivity-thermodynamics relationship, ${ }^{5,6}$ was also unexpected on the basis of the FMO model, since acetylene and ethylene have very different HOMO-LUMO gaps. Moreover, the transition structure (TS) geometries are also remarkably independent of the dipolarophile. While the distorted dipolaro-

(4) (a) Fukui, K. Angew. Chem., Int. Ed. 1982, 21, 801. (b) Fleming, I. Frontier Orbitals and Organic Chemical Reactions, Wiley: Chichester, U.K., 1976.

(5) Evans, M. G.; Polanyi, M. Trans. Faraday Soc. 1938, 34, 614.

(6) Marcus, R. A. Pure Appl. Chem. 1997, 69, 13. 
Scheme 1. The Nine 1,3-Dipoles Involved in This Work and in the Studies of Ess and Houk ${ }^{2,3}$

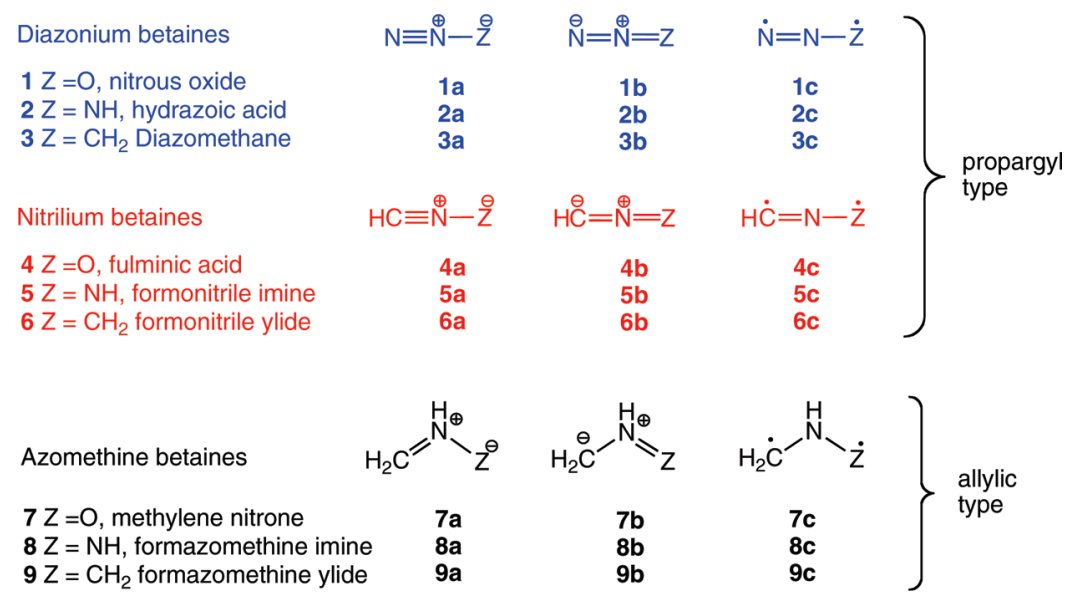

philes have, of course, different bond lengths (1.36-1.39 ̊ for ethylene vs $1.22-1.23 \AA$ for acetylene), the distorted 1,3-dipoles have quasi-similar geometries for a given 1,3-dipole, whether it adds to ethylene or to acetylene, with deviations of only 0.01 $\AA$ in bond lengths and less than $2^{\circ}$ in bending angle. This implies that the transition states for the two families of reactions have the same degrees of advancement for a given dipole, in contradiction with the Hammond postulate ${ }^{7}$ that would predict earlier (more reactant-like) transition states for the most exothermic reactions.

In order to rationalize the above results, Ess and Houk have proposed a new distortion/interaction energy model for 1,3dipolar reactivity, ${ }^{2,3}$ which decomposes the barrier into two additive components: a distortion energy, $\Delta E_{\mathrm{d}}{ }^{\ddagger}$, that is the energy required to distort the separate dipole and dipolarophile from their equilibrium geometry to their TS geometries, and the interaction energy, $\Delta E_{\mathrm{i}}^{\ddagger}$, of the two distorted fragments in the TS geometry. This model, which has also been popularized by Bickelhaupt ${ }^{8}$ and Morokuma, ${ }^{9}$ proved amazingly successful for the 18 above reactions, showing a remarkable correlation between barrier heights and distortion energies. It was further noted that the majority of the transition state distortion energy $(80 \%)$ arises from deformation of the 1,3-dipole. The distortion involves bending in all cases, which led to the prediction, confirmed by dynamic calculations, ${ }^{10}$ that the reaction should proceed more readily if the bending vibration is selectively excited.

The remarkable findings of Ess and Houk prompted us to search for the fundamental reasons behind the success of the distortion/interaction model for 1,3-dipolar cycloadditions and to try to answer a number of questions that the model leaves open. (i) Why does cycloaddition reactivity follow the HOMO-LUMO gaps of the 1,3-dipoles, according to FMO theory, but not that of the dipolarophiles? (ii) Why do the barrier heights correlate so nicely with the distortion energies, while this is not the case in many other reactions? (iii) Why are the geometries of distorted dipoles almost exactly the same, no matter if they add to ethylene or to acetylene? (iv) Finally, and

(7) Hammond, G. S. J. Am. Chem. Soc. 1955, 77, 334.

(8) (a) Bento, A. P.; Bickelhaupt, F. M. J. Org. Chem. 2008, 73, 7290. (b) de Jong, G. T.; Bickelhaupt, F. M. ChemPhysChem 2007, 8, 1170.

(9) (a) Kitaura, K.; Morokuma, K. Int. J. Quantum Chem. 1976>, 10, 325. (b) Nagase, S.; Morokuma, K. J. Am. Chem. Soc. 1978, 100, 1666.

(10) Xu, L.; Doubleday, C. E.; Houk, K. N. Angew. Chem., Int. Ed. 2009, 121, 2784.

most importantly, can one correlate the distortion energies, and therefore the barrier heights, to some quantitative properties of the reactants? Since all the 18 reactions at hand have been shown to proceed by a concerted mechanism, like the great majority of 1,3-dipolar cycloadditions, according to a general consensus, ${ }^{3,11-13}$ only the concerted TS will be investigated in this study.

Why should 1,3-dipoles behave differently from other types of reactants in elementary one-step reactions? The answer might lie in the specific nature of their electronic state. Indeed, while in the general case a reactant is described by a single Lewis structure, 1,3-dipoles are described as a combination of three structures $N \mathbf{a}, \boldsymbol{N b}$, and $N \mathbf{c}(\boldsymbol{N}=\mathbf{1 - 9})$, shown in Scheme 1, the proportions of which are expected to be dependent on the geometric distortions. Among these structures, two of them, the closed-shell zwitterionic structures $\mathbf{N a}$ and $\mathbf{N b}$, are not particularly reactive, while the singlet diradical structure $\mathrm{Nc}$, on the contrary, is extremely reactive, like all diradicals. Other Lewis structures have been shown to be minor and negligible in all cases. ${ }^{14}$

Given this multistructure character of the electronic state of 1,3-dipoles, it is clear that the reaction will be facilitated if the distortion is made in such a way that the diradical character of the 1,3-dipole increases: i.e., if the weight of its diradical structure increases. This reasoning led us to propose the following hypothesis to explain the startling results of Ess and Houk: In the process of a 1,3-dipolar cycloaddition to ethylene or acetylene, the 1,3-dipole first distorts so as to reach a reactive state that is independent of the dipolarophile; then it adds with little or no barrier to the dipolarophile, as in eqs 1 and 2 . Another aspect of this mechanism is that the reactive state is supposed to have reached some critical diradical character, the measure of which remains to be specified.

phase 1: 1,3-dipole (equilibrium) $\rightarrow$ 1,3-dipole

(reactive state) $\Delta E>0$

(11) (a) Huisgen, R. 1,3-Dipolar Cycloaddition. Introduction, Survey, Mechanism. In 1,3-Dipolar Cycloaddition Chemistry; Padwa, A., Ed.; Wiley-Interscience: New York, 1984; Vol. 1, pp 1-176. (b) Huisgen, R. Pure Appl. Chem. 1981, 53, 171.

(12) 1,3-Dipolar Cycloaddition Chemistry, Padwa, A., Ed.; Wiley-Interscience: New York, 1984; Vols. 1 and 2.

(13) Houk, K. N.; Gonzales, J.; Li, Y. Acc. Chem. Res. 1995, $28,81$.

(14) (a) Hiberty, P. C.; Leforestier, C. J. Am. Chem. Soc. 1978, 100, 2012. (b) Hiberty, P. C.; Ohanessian, G. J. Am. Chem. Soc. 1982, 104, 66. 
phase 2: 1,3-dipole (reactive state) + dipolarophile $\rightarrow$ product $\Delta E$ small

Of course, the 1,3-dipole (reactive state) is not an intermediate in the usual sense with a given lifetime. It represents a specific point on the potential surface near or on top of the reaction barrier (see section $\mathrm{C}$ in the Results and Discussion). As such, the two processes of eqs 1 and 2 represent the two phases of the 1,3-dipolar cycloaddition which possesses a concerted mechanism, as amply shown theoretically and experimentally.

This hypothesis would explain at once the similarity of the barrier heights in additions to ethylene or acetylene, the similarity of the TS geometries, and the correlation between barriers and distortion energies. It should be noted in that respect that Harcourt has been first to stress the importance of the diradical structure in 1,3-dipoles and its possible importance in 1,3-dipolar cycloadditions and coined the term "concerted diradical mechanism". ${ }^{15}$ Other authors have also discussed the electronic reorganization that occurs along the reaction coordinate of these reactions. ${ }^{16-18}$ Note that the dominant role of the distortion energy has been also stressed for other concerted reactions, such as the Diels-Alder reaction and the trimerization of ethylene, ${ }^{19}$ and for the $\mathrm{S}_{\mathrm{N}} 2$ reaction. ${ }^{20}$

The aim of this paper is to test the above hypothesis by appropriate computational means and to explore its possible manifestations in terms of a relationship between the barrier heights and some properties of the reactants in their equilibrium geometries. Indeed, if eq 1 is the effective mechanism of the reactions under study, one expects that the reactive state of eq 1 will be easier to attain if the 1,3-dipole already contains a good deal of diradical character in its ground state at equilibrium geometry. Therefore, one should find some correlation between the weights of the diradical structures in the 1,3-dipoles and the barrier heights to their cycloadditions: the larger the diradical weight, the lower should be the barrier. Moreover, another way to test the hypothesis of eq 1 would be to calculate, for each 1,3-dipole, the promotion energy that is required to go from the dipole's ground state in its equilibrium geometry to the pure diradical state in the same geometry. Once again, a correlation might be anticipated between this vertical energy gap and the barrier to cycloaddition. These two computational tests both require a computational method of valence bond (VB) type, ${ }^{21,22}$ so as to compute the weights of the individual Lewis structures and the energies of the diabatic diradical states. These will be carried out in this work by means of the "breathing-orbital

(15) (a) Harcourt, R. D. J. Mol. Struct. 1972, 12, 351. (b) Harcourt, R. D. Tetrahedron 1978, 34, 3125. (c) Harcourt, R. D. Tetrahedron 1979, 35, 901. (d) Harcourt, R. D. In Qualitative Valence Bond Description of Electron-Rich Molecules; Springer-Verlag: Berlin, 1982; Lectures Notes in Chemistry Vol. 30, p 239. (e) Harcourt, R. D.; Roso, W. Can. J. Chem. 1978, 56, 1093. (f) Harcourt, R. D.; Little, R. D. J. Am Chem. Soc. 1984, 106, 41. (g) Harcourt, R. D. J. Mol. Struct. (THEOCHEM) 1997, 398-399, 93. (h) Harcourt, R. D.; Schulz, A J. Phys. Chem. A 2000, 104, 6510. (i) Harcourt, R. D. J. Phys. Chem. A 2001, 105, 10947.

(16) Sakata, K. J. Phys. Chem. A 2000, 104, 10001

(17) Nguyen, M. T.; Chandra, A. K.; Sakai, S.; Morokuma, K. J. Org. Chem. 1999, 64, 65.

(18) Karadakov, P. B.; Cooper, D. L.; Gerratt, J. Theor. Chem. Acc. 1998, $100,222$.

(19) Ioffe, A.; Shaik, S. J. Chem. Soc., Perkin Trans. 2 1992, 2101.

(20) Mitchell, D. J.; Schlegel, H. B.; Shaik, S. S.; Wolfe, S. Can. J. Chem. 1985, 63, 1642 .

(21) Hoffmann, R.; Shaik, S.; Hiberty, P. C. Acc. Chem. Res. 2003, 36, 750 .

(22) Shaik, S.; Hiberty, P. C. A Chemist's Guide to Valence Bond Theory; Wiley-Interscience: New York, 2007. valence bond" method (BOVB), an ab initio valence bond method which has been amply tested in the past for its reliability. ${ }^{23}$

\section{Theory and Methodology}

VB Methods. Differently from MO-based methods, in which the wave functions are based on Slater determinants with delocalized MOs, VB theory uses Heitler-London-Slater-Pauling (HLSP) covalent and ionic structures as building blocks of the state functions. While it is always possible to treat all the orbitals and electrons at the VB level, here we define an "active system", which is made of the orbitals and electrons of the 1,3-dipole that are directly involved in the cycloaddition, e.g. the $\pi$ orbitals of the allylic 1,3-dipoles, or one of the $\pi$ systems in the linear 1,3-dipoles, and so on. In all the cases, the active system is made of three orbitals and four electrons, from which six VB structures can be generated in principle. However, as has been shown long ago, ${ }^{14}$ three of these VB structures are minor and can be neglected, so that we are left with only three important VB structures: $N \mathbf{a}, \mathbf{N b}$, and $N \mathbf{c}$ in Scheme 1. The electrons and orbitals of the rest of the electronic system, called a "spectator" or "inactive" system, are treated at the MO level: i.e., as optimized doubly occupied orbitals of fixed occupancies.

The VB wave function $\Psi$ of each 1,3-dipole is expressed as a linear combination of HLSP functions, here the three VB structures $\Phi_{\mathrm{a}}, \Phi_{\mathrm{b}}$, and $\Phi_{\mathrm{c}}$, corresponding to $N \mathbf{a}, N \mathbf{b}$, and $N \mathbf{c}$ :

$$
\boldsymbol{\Psi}=C_{\mathrm{a}} \boldsymbol{\Phi}_{\mathrm{a}}+C_{\mathrm{b}} \boldsymbol{\Phi}_{\mathbf{b}}+C_{\mathrm{c}} \boldsymbol{\Phi}_{\mathbf{c}}
$$

where $C_{\mathrm{K}}$ are structural coefficients.

The weights of the VB structures are defined by the Coulson-Chirgwin formula, ${ }^{24}$ eq 4 , which is the equivalent of a Mulliken population analysis in VB theory.

$$
W_{K}=C_{\mathrm{K}}^{2}+\sum_{\mathrm{L} \neq \mathrm{K}} C_{\mathrm{K}} C_{\mathrm{L}}\left\langle\Phi_{\mathrm{K}} \mid \Phi_{\mathrm{L}}\right\rangle
$$

There are several computational approaches for VB theory at the ab initio level. In the VBSCF procedure, ${ }^{25}$ both the VB orbitals and structural coefficients are optimized simultaneously to minimize the total energy. As such, the VBSCF method is nearly equivalent to the MO-based CASSCF method and takes care of the static electron correlation; however, it lacks dynamic correlation.

The BOVB method ${ }^{23}$ uses different orbitals for different VB structures. As such, the orbitals respond to the instantaneous fields of the individual VB structures rather than to an average field of all the structures. In doing so, the BOVB method accounts for part of the dynamic correlation, while leaving the wave function as compact as in VBSCF.

The BOVB method can be used at several levels of sophistication. Here we use the so-called "D-BOVB" level, ${ }^{23}$ in which the orbitals of the active system are constrained to be each localized on a single atom, while the spectator orbitals are free to delocalize over the whole molecule. Because of technical difficulties for the computation of transition energies at the TS geometries, a partially delocalized D-BOVB* level is used for the data shown in Figure

(23) (a) Hiberty, P. C.; Flament, J. P.; Noizet, E. Chem. Phys. Lett. 1992, 189, 259-265. (b) Hiberty, P. C.; Humbel, S.; Byrman, C. P.; van Lenthe, J. H. J. Chem. Phys. 1994, 101, 5969-5676. (c) Hiberty, P. C.; Shaik, S. Theor. Chem. Acc. 2002, 108, 255-272.

(24) Chirgwin, H. B.; Coulson, C. A. Proc. R. Soc., Ser. A 1950, 2, 196209.

(25) (a) Verbeek, J.; van Lenthe, J. H. J. Mol. Struct. (THEOCHEM) 1991, 229, 115-137. (b) van Lenthe, J. H. Int. J. Quantum Chem. 1991, 40, 201-210. (c) Balint-Kurti, G. G.; Benneyworth, P. R.; Davis, M. J.; Williams, I. H. J. Phys. Chem. 1992, 96, 4346-4353.

(26) (a) Song, L.; Wu, W.; Mo, Y.; Zhang, Q. XMVB: An Ab Initio Nonorthogonal Valence Bond Program; Xiamen University, Xiamen, People's Republic of China, 1999. (b) Song, L.; Mo, Y.; Zhang, Q.; Wu, W. J. Comput. Chem. 2005, 26, 514. (c) Song, L.; Song, J.; Mo, Y.; Wu, W. J. Comput. Chem. 2009, 30, 399. 
Table 1. Weights of the Various Valence Bond Structures for the Nine 1,3-Dipoles, As Calculated at the D-BOVB Level

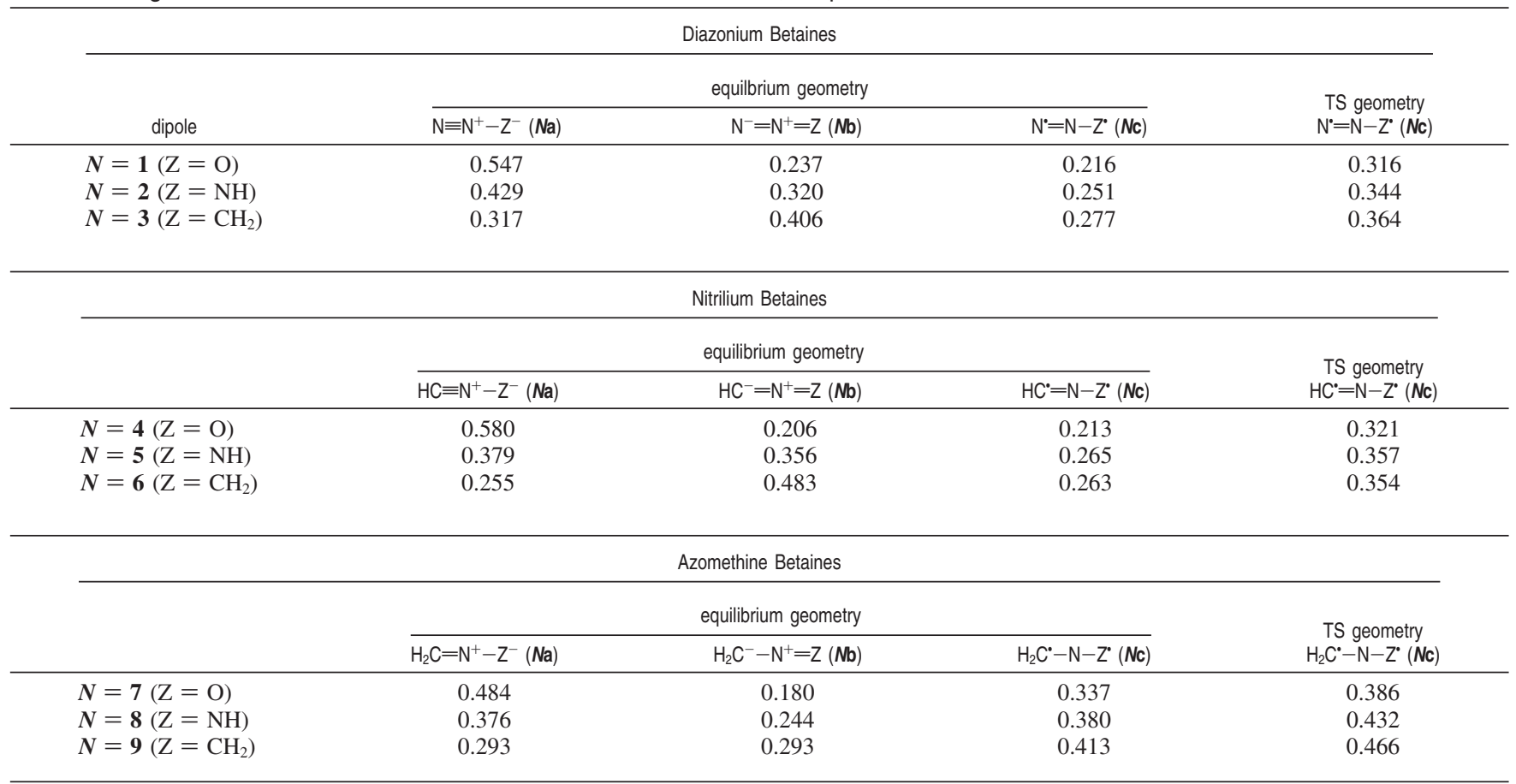

2 and for the weights at the TS geometries in Table 1. At this level, a fully localized L-BOVB calculation is first done, where all inactive pairs are localized on one atom (lone pairs) or two atoms (bonding MOs). Then, in a second step, the active orbitals are frozen while the inactive orbitals are delocalized over the whole molecule. Comparisons made on ground-state geometries show that this $\mathrm{D}-\mathrm{BOVB} *$ level gives weights almost identical with those of the full D-BOVB level as well as similar gap trends within a series.

The geometries used in this work, as well as the barrier heights that are reported in Table 1 or plotted in Figures 1 and 3, are taken from the accurate CBS-QB3(B3LYP/6-311G(d,p)) calculations of Ess and Houk, previously published in ref 3 .

The standard $6-31 \mathrm{G}^{*}$ basis set has been used throughout. Preliminary test calculations have shown that the weights of VB structures are practically unchanged if one increases the size of the basis set from 6-31G* to the cc-pVTZ basis set of triple- $\zeta$ quality. The VB calculations were done with the Xiamen VB (XMVB) package. ${ }^{25}$

\section{Results and Discussion}

A. Weights of VB Structures for the 1,3-Dipoles. The calculated weights for the different VB structures of the nine 1,3-dipoles are displayed in Table 1 . It can be seen that for each category of dipoles, the zwitterionic structures bearing a negative charge on $\mathrm{Z}$ (structures $\mathbf{N a}$ ) decrease in weight from oxide to imine to ylide, to the benefit of the other zwitterionic structure $(\mathbf{N b})$, e.g. 1a $>\mathbf{2 a}>\mathbf{3 a}, \mathbf{4 a}>\mathbf{5 a}$ and so on, in agreement with the decrease of electronegativity from $\mathrm{O}$ to $\mathrm{NH}$ to $\mathrm{CH}_{2}$. The diradical structures are far from being negligible at equilibrium geometry, with weights ranging from 0.22 to 0.41 (column 3 of the table), and even become the major VB structures for $\mathbf{8}$ and $\mathbf{9}$. As a general tendency, the diradical structures are clearly more important in the allylic dipoles (7-9) than in the propargyl ones $(\mathbf{1}-\mathbf{6})$, as was found in an earlier VB study performed at a level much lower than the present one. $^{14}$

The weights of the diradical structures in the distorted 1,3dipoles (TS geometry) are displayed in Table 1, column 4. It is immediately apparent that the diradical weights are significantly increased in the distorted geometries relative to equilibrium geometries in all cases, in agreement with the above hypothesis that the distortion serves to change the electronic structure of the dipoles to make them more reactive. It is worth noting that the relative increase of diradical weight is not the same for all dipoles. For those compounds that have a weak diradical character at equilibrium, such as nitrous oxide $\mathbf{1}$ and fulminic acid 4 , the distortion increases the diradical weight by $46 \%$ and $51 \%$, respectively, as compared to only $13 \%$ for formazomethine ylide 9, which already has a prominent diradical weight at equilibrium.

Let us now consider the relationship between barrier heights and diradical weights. In the absence of available experimental data for 1,3-dipolar cycloadditions to ethylene or acetylene in the gas phase, we will use the barrier heights calculated by Ess and Houk ${ }^{2,3}$ at the CBS-QB3 computational level, which is known to provide relative energies close to chemical accuracy. The barrier heights are plotted vs diradical weights in Figure 1, where the different families of 1,3-dipoles (diazonium, nitrilium, and azomethine betaines) are considered separately and represented in blue, red, and black, respectively. It is seen that, within each family, the barrier heights are closely related to the weights of the diradical structure in the equilibrium geometry of the 1,3-dipole, through linear relationships with excellent correlation coefficients, ranging fro 0.98 to 1.00 . Thus, as predicted by our hypothesis of a significant diradical character in the reactive state of eq 1, the lower the diradical weight, the larger the barrier. It is worth noting that formonitrile ylide $\mathbf{6}$, which was found to deviate from the qualitative correlations between barrier heights and HOMO-LUMO gaps, ${ }^{2}$ is no longer an exception and finds its place on the correlation line of nitrilium betaines: 5 and 6, which have the about same diradical weights at equilibrium, also have barrier heights that are close to each other.

B. Energy Gaps between Ground State and Pure Diradical Structure in 1,3-Dipoles. The calculated vertical transition energies between the ground states of the nine 1,3-dipoles and their pure diradical structures are displayed in Figure 2. Let us 


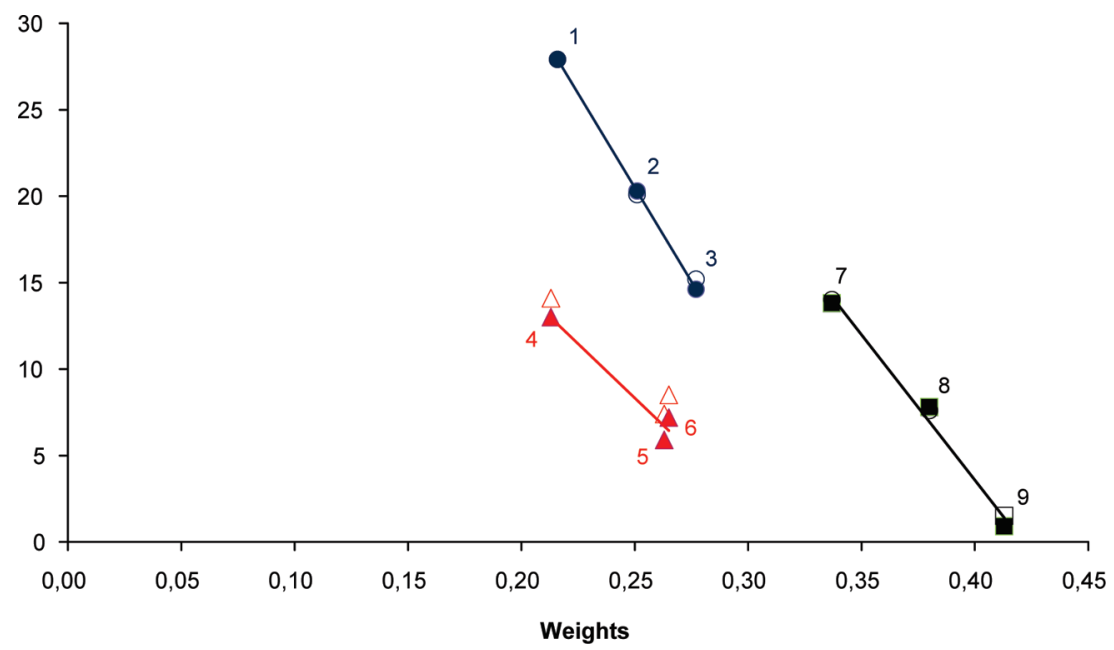

Figure 1. Plots of CBS-QB3-computed reaction barriers to cycloaddition, taken from ref 3, vs diradical weights of the 1,3-dipoles in their equilibrium geometries: (blue circles) diazonium betaines; (red triangles) nitrilium betaines; (black squares) azomethine betaines. Solid symbols indicate reactions with ethylene, and open symbols indicate reactions with acetylene.

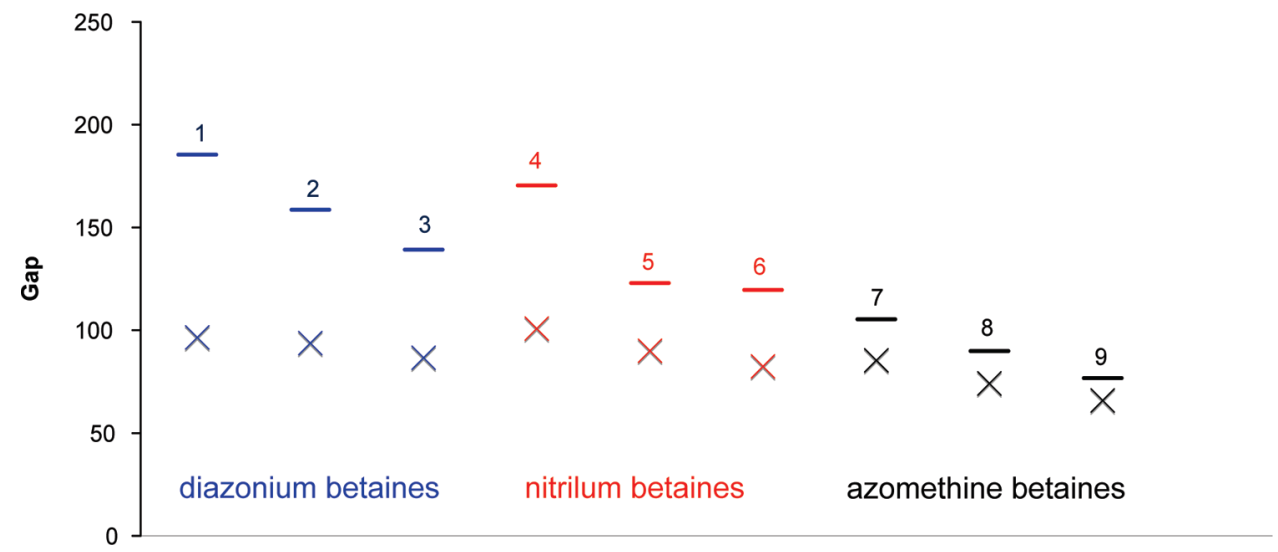

Figure 2. Vertical energy gaps ( $\mathrm{kcal} / \mathrm{mol})$ between the ground states and pure diradical states of 1,3-dipoles, in their equilibrium geometries (bars) and in their transition state geometries (crosses). Blue, red, and black symbols correspond to diazonium, nitrilium, and azomethine betaines, respectively.

consider first the energies calculated at the equilibrium geometries, represented as horizontal bars. The window of transition energies is quite large and stretches from 76 to $185 \mathrm{kcal} / \mathrm{mol}$. As expected, the transition energies are consistently smaller for the allylic dipoles (7-9) than for the propargylic ones $(\mathbf{1}-\mathbf{6})$, in agreement with the greater diradical character of the former, in terms of weights (see Table 1): the larger the weight of the diradical structure in the ground state, the smaller the energy required to reach the pure diradical state. This tendency is even clearer if one considers the dipoles family by family: the transition energies are in the order $\mathbf{1}>\mathbf{2}>\mathbf{3}$, then $\mathbf{4}>\mathbf{5} \approx \mathbf{6}$, and finally $7>8>9$ : i.e., in reverse order of the diradical weights displayed in Table 1. Figure 2 also displays the transition energies from ground state to diradical structure in the geometry of the TS, as represented by crosses. It is seen that the transition energies are consistently smaller in the distorted geometries, showing once again that the electronic states of the 1,3-dipoles are more diradical-like in the distorted geometries than at equilibrium.

Back to the properties of 1,3-dipoles at equilibrium geometries, let us now consider the relationship between the transition energies to the diradical structure and the barrier heights to cycloadditions. Figure 3 displays such a relationship for the nine 1,3-dipoles, family by family. As has been found for the diradical weights, the transition energies from ground state to diradical structures correlate very well with the barrier heights, displaying nice linear relationships with correlation coefficients equal to 0.99 or 1.00 . Once again, 6 finds its natural place on the correlation line of nitrilium betaines and is no longer an exception as in the barrier-FMO relationship., ${ }^{2,3}$

C. What is the Nature of the "Reactive State" of 1,3-Dipoles? Our hypothesis of a cycloaddition mechanism following eq 1 implies that the 1,3-dipoles do not react before they reach a certain reactive state, which is independent of the dipolarophile. Now how is this reactive state characterized, and are its characteristics transferable from one reaction to the other among the 18 reactions studied here? Up to now we only know that this reactive state has an increased diradical character relative to the corresponding 1,3-dipole in its equilibrium geometry. This diradical character can be measured in two ways: the weight of the diradical structure or the energy difference between the actual state and the pure diradical state.

One may first notice from Table 1 that the diradical weight increases more from reactant to TS when the starting diradical weight is small (e.g., 4) than when it is large (e.g., 9). The diradical weight increases indeed by $51 \%$ in $\mathbf{4}$, vs $35 \%$ in $\mathbf{5}$ and $\mathbf{6}$ and only $13 \%$ in $\mathbf{9}$, as if what mattered was to reach a critical value of the diradical weight in the TS, no matter the weight in the reactants. Of course, this critical diradical weight cannot be the same for the linear dipoles (propargylic, 1-6) 


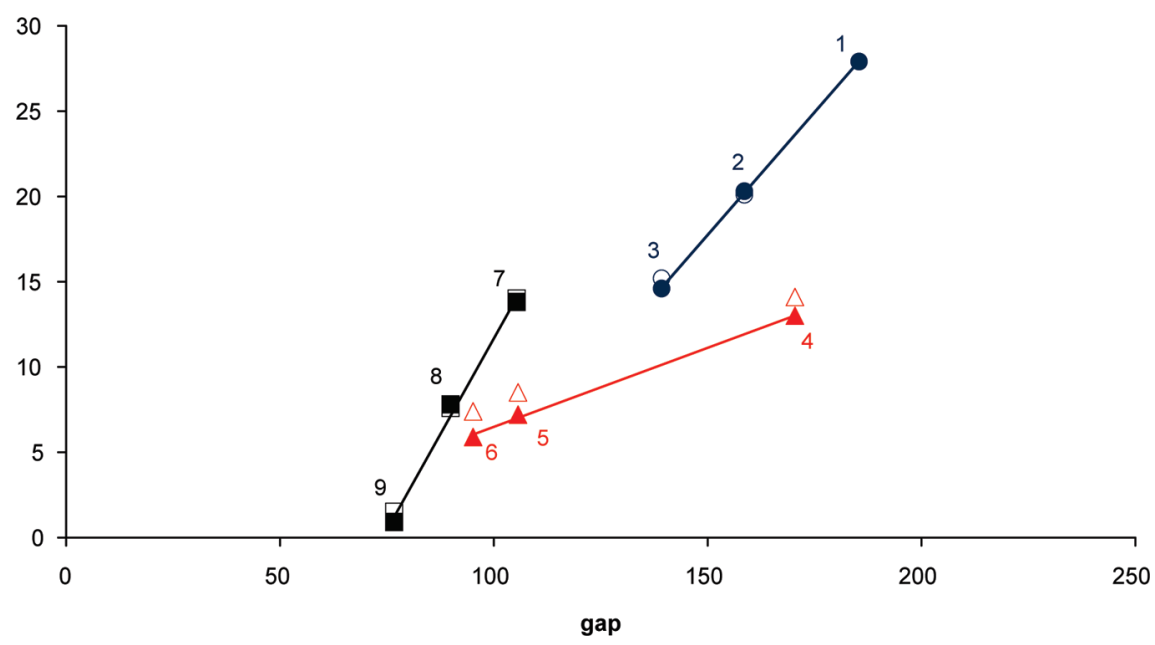

Figure 3. Plots of CBS-QB3-computed reaction barriers to cycloaddition, taken from ref 3, vs vertical energy gaps between the ground states of 1,3-dipoles and the vertical pure diradical states. The symbols are the same as those in Figure 1.

and for the bent dipoles (allylic, 7-9), which are of rather different natures. From Table 1, this critical weight can be estimated as $0.34 \pm 0.025$ for the linear dipoles and $0.426 \pm$ 0.04 for the bent dipoles. However, the best property that displays some constancy throughout the various distorted dipoles is probably to be found in the transition energies from ground state to pure diradical state. Thus, it is seen in Figure 2 that these energy gaps vary considerably from one dipole to another at the equilibrium geometries (horizontal bars in Figure 2), spanning a range of 76 to $185 \mathrm{kcal} / \mathrm{mol}$, with somewhat erratic values. In contrast, the transition energies span a much narrower range in the distorted geometries (crosses in Figure 2), from 66 to $101 \mathrm{kcal} / \mathrm{mol}$, and do not depart much from an imaginary horizontal line. Moreover, if one considers the linear and bent dipoles separately, as argued above, the range of transition energies is further reduced, to $82-101 \mathrm{kcal} / \mathrm{mol}$ in the first case $(\mathbf{1}-\mathbf{6})$ and $66-86 \mathrm{kcal} / \mathrm{mol}$ in the second one (7-9). It appears therefore that the fundamental property that characterizes the reactive state of eq 1 is a critical energy gap between this state and the pure diradical structure, and this critical energy gap amounts to $91 \pm 10 \mathrm{kcal} / \mathrm{mol}$ for propargylic dipoles and $76 \pm$ $10 \mathrm{kcal} / \mathrm{mol}$ for the allylic dipoles.

Since the reactive state is characterized by its diradical character, no matter which way the latter is quantified, it is logical to expect that the greater the diradical character in the reactant, the lower the energy required to reach the reactive state. In other words, a relationship between the barrier and the properties of the 1,3-dipoles in their equilibrium geometries is expected. This relationship exists, as has been shown above (see Figures 1 and 3). However, it is not universal: i.e., it only holds within a given family of 1,3-dipoles. The reason for that becomes clear if one considers the TS geometries of the 1,3dipoles, which can be consulted in ref 3 , and the nature of the distortion that is required to increase the diradical character in each family of dipoles. In diazonium betaines, the distortion is pure bending and amounts to $40 \pm 5^{\circ}$ on average. ${ }^{3}$ In nitrilium betaines, the bending is less important, $33 \pm 6^{\circ}$, and is accompanied by orbital rehybridization of the type $\mathrm{p} \rightarrow \mathrm{sp}^{2}$ at the $\mathrm{CH}$ site. Lastly, in azomethine betaines, the bending motion is tiny, only $9 \pm 2^{\circ}$, while rehybridization of another type, $p \rightarrow$ $\mathrm{sp}^{3}$, takes place at the $\mathrm{CH}_{2}$ site. Therefore, it is clear that, for a given diradical weight in the reactant, the energy required to reach the critical diradical weight of the reactive state will not be the same depending on whether the 1,3-dipole belongs to the diazonium, nitrilium, or azomethine family of betaines. This readily explains why the barrier/diradical-weight linear relationship can only hold within an individual family, rather than being universal. The same reasoning holds if the transition energy from ground state to pure diradical state is taken as the criterion to quantify the diradical character, as in Figure 3.

D. Relation to Other Reactivity Models. As has been seen above, neither the FMO model ${ }^{4}$ nor the reactivity-thermodynamics relationship, ${ }^{5,6}$ taken separately, can account for the fact that the barrier heights and distorted dipole geometries are practically the same, whether the dipoles add to ethylene or acetylene. However, it should be noted that the FMO model would predict higher barriers and later transition states for the addition to acetylene, since the HOMO-LUMO gap is greater in acetylene than in ethylene, leading to weakened interactions between frontier orbitals. On the other hand, the reactivity-thermodynamics relationship would predict a lower barrier height and earlier transition state for the addition to acetylene: i.e. predictions opposite to those of the FMO model. It follows that the combination of the two models would lead to the correct prediction. Of course, using a combination of two models is not really practical and would not allow semiquantitative predictions to be made, but the point we want to make is that the FMO model does not really fail for 1,3-dipolar cycloadditions.

Actually, the FMO model alone is successful, as Ess and Houk showed, ${ }^{2,3}$ if applied to the 1,3-dipoles but not to the dipolarophile, with only one exception: formonitrile ylide $\mathbf{6}$. This further supports the validity of our proposed reaction mechanism, in terms of eq 1 , in which the barrier only depends on the first formal phase, eq 1, which itself only depends on the 1,3-dipole. In this line, the connection between the FMO model and our correlations based on the diradical character is now clear: the smaller the HOMO-LUMO gap of the 1,3-dipole, the larger the weight of its diradical structure in the ground state, and the smaller the transition energy required to reach the pure diradical diabatic state. The formulation of 1,3-dipole reactivity in terms of diradical character has, however, two advantages over a formulation in terms of HOMO-LUMO gaps. (i) The calculated LUMO energy is generally quite basisset dependent, thus making it difficult to use the FMO model in a quantitative way. The calculation of the weights of valence bond structures does not generally have this inconvenience. (ii) The diradical weights and/or transition energies are calculated at the correlated-electron level, while the FMO model considers 
the 1,3-dipoles at the one-electron level. This computational level, which is devoid of electron correlation, does not describe very well molecules that have a multireference character, particularly when the diradical character is important.

Our calculations support and complete the distortion/interaction energy model of Ess and Houk, which is based on the fact that the barrier heights for 1,3-dipolar cycloadditions to ethylene or acetylene only depend of the properties of the 1,3-dipoles and are closely related to their distortions in the geometries of the transition states. ${ }^{2,3}$ In addition, the present work elucidates the reason why the distortion of the 1,3-dipole is the key event that allows the reaction to take place. If the distortion merely consisted of orienting the cycloaddends so as to favor the overlap of the newly interacting orbitals, the distorted geometries would depend on the nature of the dipolarophiles, since acetylene has a much shorter bond length than ethylene. On the other hand, if, as our calculations suggest, the role of the distortion is to transform the electronic state of the 1,3-dipole so as to make it more reactive, then identical distortions are expected for additions to ethylene and acetylene, which is indeed the case.

E. Conclusion. This work has been stimulated by the recent findings of Ess and Houk that the barrier heights and transition structure geometries are identical for cycloadditions to ethylene or acetylene $\mathrm{e}^{2,3}$ and only depend on the distortion of the 1,3dipole. In order to explain these findings, we propose a reaction mechanism in which the 1,3-dipole first distorts so as to reach a reactive electronic state that has a significant diradical character, which then adds with little or no barrier to the dipolarophile. This mechanism is supported by the calculated properties of the distorted 1,3-dipoles, which share some common features from one reaction to the other in terms of diradical weights and especially in terms of transition energies. Thus, the window of transition energies from ground state to diradical states is fairly narrow in the distorted dipoles, which allows a definition of the "reactive state" of 1,3-dipoles that must be reached before the cycloaddition takes place: the reactive state is a state having enough exalted diradical component so that its transition energy to the pure diradical state is reduced to $91 \pm 10 \mathrm{kcal} / \mathrm{mol}$ for propargyl dipoles and $76 \pm 10 \mathrm{kcal} / \mathrm{mol}$ for the allylic dipoles.

The present work also evidences a clear correlation between the diradical character of nine 1,3-dipoles, belonging to the families of diazonium, nitrilium, and azomethine betaines, and their reactivity to cycloaddition to ethylene or acetylene.
Whether the diradical character is measured as the weight of the diradical structure at equilibrium or as the energy gap between the ground state of the 1,3-dipole and its diradical diabatic state, the correlations with the barrier heights are excellent, with correlation coefficients ranging from 0.98 to 1.00 . Of course, owing to structural differences between the different families of 1,3-dipoles, the correlation is not general but only holds within a given family. Be this as it may, all the 18 reactions under study display a clear relationship between the barrier and a property of the reactants that can be quantitatively estimated. Finally, both the nature of the reactive state and the above correlations support the early assumption of Harcourt that the diradical valence bond structure of 1,3-dipoles should play an important role in 1,3-dipolar cycloadditions. ${ }^{15}$

An important point to note is that the diradical character of the reactive state, albeit important, is still very far from that of a pure diradical state. Therefore, the present results can in no way be interpreted as a support either for a diradical mechanism or for a stepwise reaction being as good as the concerted reaction. Finally, it must be kept in mind that the 18 reactions investigated here are cycloadditions in which the dipolarophiles are not polarized by substituents and are of comparable electron richness. Thus, as noted by Ess and Houk in terms of the distortion/interaction energy model, "when distortion energies are approximately the same for a series of different dipolarophiles, interactions energies (primarily FMO interactions) vary and become the controlling factor". ${ }^{3}$ Moreover, the interaction energies will be widely different on comparison of the transition states of electron-rich and electron-poor alkenes. Therefore, it is probable that our correlations between barrier heights and diradical character only apply for dipolarophiles of comparable richness. The influence of asymmetric substituent effects on the model is also unknown for the moment. However, in any case, it is clear that the diradical character of 1,3-dipoles is an important factor that favors 1,3-dipolar cycloaddition reactions.

Acknowledgment. We are grateful to Professor W. Wu for making his new version of the XMVB program available to us. 